\title{
Bicuspid Aortic Valve Morphology And Hemodynamics By Same-Day Echocardiography And Cardiac MRI
}

Jeesoo Lee ( $\sim$ jeesoolee@northwestern.edu )

Northwestern University https://orcid.org/0000-0002-3301-6469

Nadia El Hangouche

Northwestern Memorial Hospital

Ashitha Pathrose

Northwestern University

Gilles Soulat

Northwestern University

Alex J. Barker

University of Colorado Anschutz Medical Campus

James D. Thomas

Northwestern Memorial Hospital

Michael Markl

Northwestern University

\section{Research Article}

Keywords: Bicuspid aortic valve, Echocardiography, 4D flow MRI, Aortic valve morphology, Aortic valvular hemodynamics.

Posted Date: January 11th, 2022

DOI: https://doi.org/10.21203/rs.3.rs-1235571/v1

License: (c) (1) This work is licensed under a Creative Commons Attribution 4.0 International License. Read Full License

Version of Record: A version of this preprint was published at The International Journal of Cardiovascular Imaging on March 16th, 2022. See the published version at https://doi.org/10.1007/s10554-022-02593-0. 


\section{Abstract}

PURPOSE: This study investigated the impact of bicuspid aortic valve (BAV) on valve morphology and motion as well as proximal and aortic hemodynamics using a same-day echocardiography and cardiac MRI.

METHODS: Transthoracic echocardiography, two-dimensional cine MRI of the aortic valve, and aortic 4D flow MRI were performed on the same day in 9 normofunctional BAV patients (age $=41 \pm 12,3$ female), 4 BAV with moderate to severe aortic stenosis (AS) (age $=63 \pm 5,1$ female), and 36 healthy tricuspid aortic valve controls (age $=52 \pm 10,21$ female). Valve opening and closing timings and transvalvular peak velocity were measured using B-mode and Doppler echocardiogram, respectively. Valve orifice morphology at fully-opened state was characterized using cine MRI. Ascending aortic (AAo) wall shear stress (WSS) was measured using 4D flow MRI data.

RESULTS: Valve motion timings were similar between BAV and controls. BAV was associated with increased orifice aspect ratio ( $1.44 \pm 0.11$ vs. $1.10 \pm 0.13, P<0.001)$, transvalvular peak velocity $(1.5 \pm 0.3$ vs. $1.2 \pm 0.2 \mathrm{~m} / \mathrm{s}, \mathrm{P}<0.001)$ and maximum AAo WSS (1.62 \pm 0.31 vs. $0.91 \pm 0.24 \mathrm{~Pa}, \mathrm{P}<0.001)$. Increased orifice aspect ratio was associated with the increase in transvalvular peak velocity $(r=0.80, P<0.0001)$ and maximum AAo WSS $(r=0.83, P<0.0001)$. Transvalvular peak velocity was also positively correlated with maximum AAo WSS $(r=0.83, P<0.0001)$.

CONCLUSION: A same-day echo and MRI imaging allows for comprehensive assessment of the impact of aortic valve disease on valve function and hemodynamics. In this pilot application to BAV, we found increased orifice aspect ratio may be responsible for increased transvalvular peak velocity and maximum AAo WSS.

\section{Introduction}

Bicuspid aortic valve (BAV) is the most common congenital cardiovascular defect that occurs in $1-2 \%$ of the general population in the United States.[1] BAV is associated with a wide range of complications such as valvular stenosis and regurgitation and aortic aneurysm and dissection.[2, 3] Progression to secondary complications is thought to be a result of genetic defects in vascular connective tissue.[4] However, additional evidence suggests that BAV can result in abnormal ascending aortic (AAo) flow associated with the development of aortopathy, even in patients without aortic valve stenosis (AS).[5-8]

Four-dimensional (4D) flow MRI has played an instrumental role in detecting valve-mediated abnormal aortic flow (e.g., helical flow patterns, eccentric valvular outflow jet patterns and skewed velocity profile) in BAV patients.[6-9] Wall shear stress (WSS), which represents the viscous friction force exerted on the aortic wall by blood flow, has been regarded as an important flow metric associated with vascular remodeling.[10] Analysis of aortic WSS by 4D flow MRI has shown asymmetric and elevated WSS in the AAo of BAV patients.[11-13] In addition, recent studies provide evidence that elevated WSS can be associated with degeneration of the aortic wall (elastic fiber thinning on histopathology).[11, 14] However, 
4D flow MRI has insufficient spatial and temporal resolution to accurately assess BAV morphology and function, potentially important physiologic mechanisms implicated in AAo flow and WSS changes. The impact of valve metrics such as opening and closing dynamics, valve orifice shape, and near-valve flow on changes in AAo WSS in BAV is thus not fully understood.

This study presents a multimodality approach that combines echocardiography (echo), 2D cine MRI, and 4D flow MRI to investigate in detail aortic valve morphology, valve motion, as well as near-valve flow and downstream AAo WSS affected in BAV patients compared to healthy subjects with normal tricuspid aortic valve (TAV). The specific objectives were 1) to assess dynamic characteristics of valve leaflet opening and closing motion and aortic valvular flow using echo, 2) to assess morphological characteristics of valve orifice shape using cine MRI, and 3) to assess downstream 3D WSS using aortic 4D flow MRI. We hypothesized that this combined echo and MRI approach can detect the relationships between BAV morphology, valve motion dynamics, near-valve and downstream aortic hemodynamics.

\section{Methods}

\section{STUDY COHORT}

A total of 13 BAV patients and 36 healthy volunteers with TAV (age $=52 \pm 10$, female $=20)$ were prospectively enrolled for same-day transthoracic echo and cardiac MRI, including 4D flow MRI. Based on American Society of Echocardiography guidelines[15], BAV patients were divided into two subgroups with and without echocardiography proven aortic valve stenosis (AS): 9 BAV patients without AS and 4 BAV patients with AS (2 moderate-to-severe and 2 severe). Demographic characteristics of each cohort are summarized in Table 1. The study was approved by our institutional review board and informed consent was obtained from all participants. 
Table 1

Patient demographics

\begin{tabular}{|c|c|c|c|c|c|c|c|}
\hline & \multicolumn{2}{|l|}{ BAV } & \multicolumn{2}{|c|}{ BAV AS } & \multicolumn{2}{|c|}{ Control } & \multirow[t]{2}{*}{ P-value } \\
\hline $\mathrm{n}$ (female) & $9(3)$ & & $4(1)$ & & $36(21)$ & & \\
\hline Age & 41 & \pm 12 & 63 & \pm 5 & 52 & \pm 10 & 0.10 \\
\hline Body Surface Area [m²] & 1.92 & \pm 0.25 & 1.97 & \pm 0.14 & 1.90 & \pm 0.25 & 0.86 \\
\hline Systolic Pressure [mmHg] & 123.0 & \pm 14.8 & 129.3 & \pm 6.8 & 123.0 & \pm 26.3 & 0.50 \\
\hline Diastolic Pressure [mmHg] & 74.8 & \pm 10.3 & 73.5 & \pm 11.0 & 76.8 & \pm 12.9 & 0.84 \\
\hline Ejection Fraction [\%] & 59.0 & \pm 5.0 & 56.3 & $\pm 5.0^{\dagger}$ & 62.0 & \pm 4.5 & 0.006 \\
\hline BAV fusion type & $\mathrm{LR}=8$ & $N=1$ & $\mathrm{LR}=4$ & & Tricusp & & \\
\hline Aortic stenosis grade & None & & $M S=2$ & $S=2$ & None & & \\
\hline \multicolumn{8}{|c|}{ † denotes a significant difference compared the control group $(P<0.017)$. } \\
\hline $\begin{array}{l}\text { Abbreviations: BAV (bicuspi } \\
\text { (severe). LR (Left-Right), RN }\end{array}$ & $\begin{array}{l}\text { itic val } \\
\text { ht-Non }\end{array}$ & $\begin{array}{l}\text { ), AS (ao } \\
\text { ronary) }\end{array}$ & c stenos & ),$M S($ & derate $t$ & severe) a & \\
\hline
\end{tabular}

\section{DATA ACQUISITION - MRI}

All MRI exams were conducted on a 1.5T or 3T MRI system (1.5T Aera, Avanto and 3T Skyra, Siemens Healthineers AG, Erlangen, Germany). The MRI protocol included 2D steady-state free precession cine MRI for imaging of the aortic valve and free-breathing 4D flow MRI covering the thoracic aorta. For cine MRI, the imaging plane was placed across the valve leaflets seen on cardiac 3-chamber and left ventricular outflow tract view images and signals were acquired during breath-hold at end inspiration with following pulse sequence parameters: echo time $=1.2-3.3 \mathrm{~ms}$, flip angle $=18-83^{\circ}$, temporal resolution $=24.6-$ $57.2 \mathrm{~ms}$, bandwidth $=400 / 930 \mathrm{~Hz} /$ pixel, field of view $=270-420 \times 244-420 \times 6$ (slice thickness) $\mathrm{mm}^{3}$ with in-plane spatial resolution $=1.4-2.2 \times 1.3-2.4 \mathrm{~mm}^{2}$. For 4D flow MRI, a sagittal oblique orientation was used as an imaging volume with the use of a prospective or retrospective electrocardiogram gating and a navigator for respiratory motion compensation. Pulse sequence parameters were as follows:

Sagittal oblique field of view $=255-315 \times 340-420 \times 68-97 \mathrm{~mm}^{3}$, spatial resolution $=2.1-2.6 \times 2.1-2.6$ $\times 2.4-3.7 \mathrm{~mm}^{3}$, echo time $=2.3-2.5 \mathrm{~ms}$, flip angle $=7 / 15^{\circ}$, temporal resolution $=38.4-40.6 \mathrm{~ms}$, bandwidth $=455 / 460 \mathrm{~Hz} /$ pixel, encoding velocity (venc) $=150-350 \mathrm{~cm} / \mathrm{s}$ along all three directions and total scan time $=806 \pm 255 \mathrm{~s}$.

\section{DATA ACQUISITION - ECHO}


All echo exams following a standard-of-care protocol were conducted using an echocardiography scanner (Vivid E95, GE Healthcare, Horten, Norway) and a transducer for adult cardiac imaging (M5Sc-D 1.4-4.6 MHz). In each set of echocardiograms of a subject, parasternal long-axis and short-axis B-mode images that captured aortic valve motion was used to assess aortic valve motion and continuous-wave Doppler velocity spectrum of flow through the aortic valve was used to assess near-valve flow dynamics.

\section{DATA ANALYSIS - ECHOCARDIOGRAPHY}

Echo data post-processing and measurements were performed using EchoPAC (v2.02, GE Healthcare, Horten, Norway). Valve leaflet motion was analyzed by measuring the timings of three leaflet motion phases: valve rapid opening time (VROT), valve slow closing time (VSCT) and valve rapid closing time (VRCT) suggested previously by De Paulis et al.[16] and Leyh et al.[17] They quantified the timings based on an aortic valve motion diagram (Figure 1A) seen on aortic valve M-mode echocardiogram. However, we noted that M-mode often fails to visualize all of the three phases due to the use of a fixed single beamline. In this study, we used parasternal long or short-axis aortic valve two B-mode echocardiogram to reconstruct the valve motion diagram B-mode echocardiogram (Figure 1B and C). The measurement procedure was as follows: 1) Place an interrogation line across the aortic valve leaflets, 2) extract B-mode signals along the line and plot them over time (Figure 1C), 3) measure the duration of each phase observable in the plot. If there is a missed phase in the diagram, the position of the interrogation line was adjusted and the step 2) and 3) were repeated. For near-valve flow dynamics characterization, peak velocity and time-to-peak velocity (TTP) of transvalvular flow were measured as a peak point and a duration between the inception of velocity increase to the peak point, respectively, as described in Figure

2. All timings were divided by $\sqrt{\text { RRinterval }}$ according to the Bazett's formula to minimize the effects of heart rate variability on the timing measurements.[18]

\section{DATA ANALYSIS - 2D CINE MRI}

Valve morphology was characterized based on 2D cine MRI images at the level of the aortic valve. The orifice and aortic sinus region at mid-systole (largest orifice area) were manually contoured (Figure 3A) and used to quantify the following morphological parameters (Figure 3B):

- Relative orifice area: orifice area divided by the area of aortic sinus

- Orifice aspect ratio: ratio of the long-axis $\left(d_{l}\right)$ vs. short-axis diameter $\left(d_{s}\right)$. The short axis was measured based on a line from a commissural point of a conjoined leaflet on the sinus to the midpoint of the opposite leaflet. The long axis diameter, $d_{l}$, was automatically calculated based on the formula of ellipse area

$$
\left(d_{l}=4 \cdot \text { orifice area } /\left(\pi d_{s}\right)\right) \text {. }
$$

\section{D FLOW MRI - WALL SHEAR STRESS}

4D flow MRI datasets were preprocessed for phase offset correction, noise masking, and velocity antialiasing as described previously[19] using in-house MATLAB (R2018b, Mathworks Inc., Natick, USA) code. 
The thoracic aorta region within the 4D flow MRI dataset was automatically identified using a deep learning approach described previously[20] and was used to create a mask to remove velocity vectors outside of the aorta. Peak systole was defined as the time point in systole when the average velocity magnitude in the aorta was the highest. Peak-systolic aortic WSS on the aorta surface was calculated by using a previously developed method.[21] Briefly velocity gradient vectors were calculated at the surface of the aorta 3D segmentation mask and multiplied the dynamic viscosity of blood (3.2 cP) to calculate WSS vectors at the aorta wall. Figure 4 shows maximum intensity projection images of 3D aortic WSS magnitude at peak systole for a subject in each cohort. Median WSS values in the ascending aorta were quantified by a manually defined region-of-interest from the sinus of valsalva to the onset of the brachiocephalic artery. In addition, maximum WSS was determined as the mean of the $5 \%$ highest WSS values.

\section{STATISTICAL METHODS}

Numerical data are reported in mean \pm standard deviation or median \pm interquartile depending on data normality determined by the Lilliefors test. Differences between the three groups were evaluated using one-way analysis of variance or Kruskal-Wallis test. When the difference between groups was statistically significant $(\mathrm{P}<0.05)$, pair-wise difference was tested using two-tailed unpaired t-test (normal distribution) or Wilcoxon rank sum test. Bonferroni correction was used to adjust a statistical significance level for multiple comparisons as $\mathrm{P}<0.017$. Pearson correlation coefficients were calculated to investigate associations between valve morphology, valve leaflet motion timings and hemodynamic parameters. Correlation analysis was performed with datapoints from 1) all groups and 2) BAV and healthy TAV controls (BAV AS excluded).

\section{Results}

\section{STUDY COHORT}

Patient demographics are summarized in Table 1. Age, body surface area, systolic blood pressure, diastolic blood pressure between all three groups were not statistically significantly different. BAV AS patients had lower ejection fraction than TAV controls ( $56.3 \pm 5.0$ vs. $62.0 \pm 4.5, P<0.007)$.

Echocardiography-based valve leaflet motion timings could not be analyzed for cases when image quality was insufficient to identify the aortic valve and/or when out-of-plane valve movement made the aortic valve disappear during the valve closing phase. VROT was unobtainable from 2 BAV AS patients and 8 healthy controls. VSCT and VRCT could not be calculated in 1 BAV patient, 2 BAV AS patients and 13 TAV controls.

\section{ECHOCARDIOGRAPHIC MEASUREMENTS - VALVE LEAFLET MOTION AND NEAR-VALVE FLOW DYNAMICS}

As summarized in Table 2, VROT, VSCT, VRCT were similar between all three groups. Faster transvalvular peak velocity was observed for BAV patients compared to TAV controls $(1.5 \pm 0.3 \mathrm{~m} / \mathrm{s}$ vs. $1.2 \pm 0.2 \mathrm{~m} / \mathrm{s}, \mathrm{P}$ 
$<0.001)$ which further increased in BAV AS patients, as expected $(4.0 \pm 0.8 \mathrm{~m} / \mathrm{s}, \mathrm{P}<0.001 \mathrm{vs}$. BAV and healthy controls). Transvalvular TTP was similar for BAV and healthy controls (71 $\pm 5 \mathrm{vs}$. $77 \pm 15 \mathrm{~ms}, \mathrm{P}=$ 0.252). Delayed TTP was noted in BAV AS patients (124 $\pm 24 \mathrm{~ms}, \mathrm{P}<0.001 \mathrm{vs}$. BAV and TAV controls).

Table 2

Summary of echocardiographic and MRI measurements

\begin{tabular}{|c|c|c|c|c|c|c|c|}
\hline & \multicolumn{2}{|l|}{ BAV } & \multicolumn{2}{|c|}{ BAV AS } & \multicolumn{2}{|c|}{ Control } & \multirow[t]{2}{*}{ P-value } \\
\hline Echo & & & & & & & \\
\hline Valve rapid opening [ms] & 31 & \pm 11 & 35 & \pm 7 & 31 & \pm 9 & 0.74 \\
\hline Valve slow closing [ms] & 267 & \pm 12 & 267 & \pm 30 & 267 & \pm 21 & 0.97 \\
\hline Valve rapid closing [ms] & 34 & \pm 8 & 37 & \pm 10 & 38 & \pm 11 & 0.40 \\
\hline Transvalvular peak velocity [m/s] & 1.5 & $\pm 0.3^{\dagger \neq}$ & 4.0 & $\pm 0.8^{\dagger}$ & 1.2 & \pm 0.2 & $<0.001$ \\
\hline Transvalvular flow TTP [ms] & 71 & $\pm 5^{\ddagger}$ & 125 & $\pm 22^{\dagger}$ & 77 & \pm 15 & $<0.001$ \\
\hline \multicolumn{8}{|l|}{ 2D cine MRI } \\
\hline Relative orifice area [\%] & 42.5 & $\pm 4.6^{\ddagger}$ & 16.5 & $\pm 2.7^{\dagger}$ & 48.4 & \pm 6.8 & $<0.001$ \\
\hline Orifice aspect ratio & 1.44 & $\pm 0.11^{\dagger \ddagger}$ & 5.99 & $\pm 4.43^{\dagger}$ & 1.10 & \pm 0.13 & $<0.001$ \\
\hline \multicolumn{8}{|l|}{ 4D flow MRI } \\
\hline Max AAo WSS [Pa] & 1.62 & $\pm 0.31^{\dagger \ddagger}$ & 2.90 & $\pm 0.76^{\dagger}$ & 0.91 & \pm 0.24 & $<0.001$ \\
\hline Median AAo WSS [Pa] & 0.77 & $\pm 0.21^{\dagger}$ & 0.92 & $\pm 0.23^{\dagger}$ & 0.60 & \pm 0.15 & $<0.001$ \\
\hline
\end{tabular}

MRI MEASUREMENTS - VALVE MORPHOLOGY

Relative orifice area was slightly smaller in BAV patients $(42.5 \pm 4.6 \%)$ than TAV controls $(48.4 \pm 6.8 \%, \mathrm{P}<$ 0.022 ), but not statistically significant (Table 2). BAV AS patients had the smallest relative orifice area $(16.5 \pm 2.7 \%, P<0.001$ vs. BAV and healthy controls) as expected. Orifice aspect ratio was significantly different between all groups. The orifice aspect ratio was close to unity in TAV controls (1.10 \pm 0.13$)$. BAV patients had higher aspect ratio $(1.44 \pm 0.11, P<0.016)$ which further increased with AS but with greater variance $(5.99 \pm 4.43, P=0.001$ vs. $B A V$ and $P<0.001$ vs. healthy controls).

\section{MRI MEASUREMENTS - AORTIC HEMODYNAMICS}

An example of WSS distribution along the aorta representing each cohort is illustrated in Figure 4 using maximum intensity projection along the anterior to posterior direction. Local high WSS regions were 
noted in the ascending aorta of BAV and BAV AS patients, whereas TAV controls have overall lower WSS. As shown in Table 2, maximum AAo WSS was significantly different between all three groups. TAV controls demonstrated the lowest maximum AAo WSS (0.91 $\pm 0.24 \mathrm{~Pa})$. BAV patients demonstrated higher maximum AAo WSS $(1.62 \pm 0.31 \mathrm{~Pa}, \mathrm{P}=0.0013)$ which increased further with AS $(2.90 \pm 0.76 \mathrm{~Pa}$, $P=0.002$ vs. BAV and $P<0.001$ vs. TAV controls). A similar trend was observed for median AAo WSS but the difference between BAV and BAV AS was not statistically significant.

\section{RELATIONSHIPS BETWEEN VALVE DYNAMICS, MORPHOLOGY AND AORTIC HEMODYNAMICS}

Relationships between morphological and hemodynamic parameters are summarized in Table 3. Significant strong correlations were found between increased relative valve orifice area and reduced transvalvular peak velocity $(r=-0.77, P<0.0001)$, between larger orifice aspect ratio and higher transvalvular peak velocity $(r=0.80, P<0.0001)$ and between larger orifice aspect ratio and increased maximum AAo WSS $(r=0.79, P<0.0001)$. As expected by moderate to severe AS status in the BAV AS group, orifice aspect ratio, peak velocity and WSS were far off from BAV and control groups as shown in Figure 5, possibly driving the correlations. When BAV AS group was excluded to remove the impact of AS on the analysis, moderate correlations remained between orifice aspect ratio and transvalvular peak velocity $(r=0.43, P=0.003)$ and maximum AAo WSS $(r=0.38, P=0.010)$ as shown in Figure $5 A$ and $B$, respectively. No significant associations were found between valve leaflet motion timings and valve morphology. Relationships between hemodynamic parameters are summarized in Table 4. A strong positive association was found between maximum AAo WSS and transvalvular peak velocity $(r=0.83, P$ $<0.0001$ ) which remained significant when BAV AS patients were excluded $(r=0.51, P<0.001)$ (Figure $5 C)$.

Table 3

Correlation analysis between valve morphology and hemodynamic parameters.

\begin{tabular}{|lll|}
\hline & Relative orifice area & Orifice aspect ratio \\
\hline Transvalvular flow peak velocity & $r=-0.77, P<0.0001$ & $r=0.80, P<0.0001$ \\
\hline Transvalvular flow TTP & $r=-0.57, P<0.0001$ & $r=0.58, P<0.0001$ \\
\hline Maximum AAo WSS & $r=-0.64, P<0.0001$ & $r=0.79, P<0.0001$ \\
\hline Median AAo WSS & Not Significant & $r=0.54, P<0.0001$ \\
\hline Abbreviation: TTP (time-to-peak velocity), WSS (wall shear stress), AAo (ascending aorta). \\
\hline
\end{tabular}


Table 4

Correlation analysis between near-valve hemodynamic parameters and ascending aortic wall shear stress.

\begin{tabular}{|lll|}
\hline & Maximum AAo WSS & Median AAo WSS \\
\hline Transvalvular peak velocity & $r=0.83, P<0.0001$ & $r=0.49, P<0.001$ \\
\hline Transvalvular TTP & $r=0.54, P<0.0001$ & $r=0.35, P=0.013$ \\
\hline Abbreviation: TTP (time-to-peak velocity), WSS (wall shear stress), AAo (ascending aorta). \\
\hline
\end{tabular}

\section{Discussion}

This pilot study demonstrated the utility of same-day echo and cardiac MRI for comprehensive characterization of BAV and associated hemodynamics. Superior spatiotemporal resolution of echo was advantageous for evaluating rapid opening and closing valve leaflet motion and near-valve flow while superior image contrast in 2D cine MRI and full volumetric thoracic aorta coverage of 4D flow MRI were advantageous for characterizing BAV morphology and 3D downstream hemodynamics. The same-day acquisition minimized potential variation in physiologic conditions between the two modalities. Despite the small number of BAV patients, we found significant differences in valve morphology (orifice aspect ratio), and hemodynamics near the valve (transvalvular peak velocity) and in the downstream (ascending aortic WSS) in BAV patients compared to healthy controls with TAV. Furthermore, moderate to strong correlations were observed between orifice aspect ratio by 2D cine MRI, transvalvular peak velocity by echo and ascending aortic WSS by 4D flow MRI.

Near-valve flow characterized using echo demonstrated significantly elevated transvalvular peak velocity in normally functioning BAV patients. This finding has been documented in a few studies[22, 23], but its cause has not been well understood. We found that increased peak velocity was associated with the orifice aspect ratio. This relationship may be explained by an elliptical orifice providing a smaller jet flow area at the location of its narrowest section (i.e., vena contracta, where peak velocity occurs) compared to a circular orifice for a given orifice area, as shown by prior in-vitro studies.[24, 25] Flachskampf et al. have further demonstrated that the vena contracta area reduces as the orifice aspect ratio increases.[25] In addition, acceleration of transvalvular flow assessed by transvalvular TTP was maintained despite the increase in peak velocity in normal BAV compared to TAV controls. Previous studies have reported that a prolonged TTP is found in severe AS patients and is associated with poor prognosis. $[26,27]$ In line with the studies, we observed a prolonged TTP in BAV AS patients suggesting that BAV might not alter temporal near valve flow characteristics unless it has progressed into AS.

The observation of elevated AAo WSS in normally functioning BAV patients with further increase in BAV AS patients is a common finding in previous literature. $[6,11,23,28]$ Increased flow derangement manifested as helical flow pattern, and eccentric and skewed flow toward the wall has been shown to contribute to the increase in AAo WSS in BAV patients.[5-7] In addition to previous findings, we observed that transvalvular peak velocity is associated with increased local AAo WSS (i.e., maximum WSS). The 
association with peak velocity may be a direct consequence since a faster valvular flow velocity is likely to create a greater velocity gradient on the wall elevating WSS. Furthermore, BAV has been shown to demonstrate higher flow displacement in the ascending aorta.[6] The skewed velocity profile would induce a greater velocity gradient on the aortic surface closer to fast velocities and thus increasing WSS locally, as illustrated in Figure 4. We also noted maximum AAo WSS increased with increased orifice aspect ratio. The larger aspect ratio of the valve orifice may account for the faster transvalvular peak velocity causing the elevation in maximum AAo WSS, as discussed in the previous paragraph.

This study was the first attempt to characterize temporal BAV leaflet motion. We found similar valve motion timings between the groups implying that abnormal leaflet structure due to leaflet fusion might not significantly impact the opening and closing characteristics of the valve. The change in aortic valve opening and closing timings have been observed previously in patients who underwent a valve-sparing procedure with different techniques (David or Yacoub) $[17,29]$ or implanted with different aortic root prosthesis[16] which produced different aortic root shape and distensibility. Previous studies based on the observations in dogs suggested that expansion of the aortic root due to an increased left ventricular pressure at the beginning of systole triggers the valve to open before the onset of aortic forward flow.[30, 31] A study based on in vitro observation suggested a vortex trapped in the sinus activates the valve closing.[32] In light of these previous studies, valve leaflet motion would be primarily affected by structural abnormalities in the valve surrounding, not the valve itself. It should be noted that the temporal resolution of B-mode echocardiogram used in this study was approximately $10 \mathrm{~ms}$ which might have limited the precision of rapid opening and closing timing measurements compared to a prospective $\mathrm{M}$ mode (1-2 ms) used previously.[16, 17] However, more subjects were analyzable (39 vs. 16 out of total 49 subjects) using B-mode that enabled retrospective adjustment of valve leaflet motion interrogation line.

A major limitation of this study is the small number of patients and the left and right leaflet fusion was predominant except for one patient with right and non-coronary fusion. Another limitation is that the ascending aorta size was not controlled in this study which would impact the distribution and magnitude of WSS.[5, 6] Lastly, the BAV AS group consisted of mostly severe AS patients whose valve morphology and flow characteristics may be dominated by AS rather than BAV. However, within this limited cohort, we were still able to observe some significant relationships between valve morphology and aortic hemodynamics. Future work is warranted to confirm the study findings in a larger BAV cohort with controlled aortic size and BAV phenotype.

In conclusion, by exploiting the unique strengths of echo and MRI, a same-day echo and cardiac MRI can serve as a useful tool for a comprehensive evaluation of valve morphology and motion, and near-valve and distal 3D hemodynamics. In this pilot application to BAV, we found increased orifice aspect ratio of BAV may be responsible for increased transvalvular peak velocity and maximum AAo WSS. This multimodality approach could be expaned to various valvular heart diseases to identify temporal and spatial characteristics of valve morphology and associated hemodynamics.

\section{Statement \& Declarations}




\section{ACKNOWLEDGEMENTS}

This study was supported by NIH R01HL115828 and R01HL133504. We would also like to thank General Electric, Inc., and the Irene D. Pritzker foundation for their financial and equipment support for this study.

\section{FUNDING}

This study was supported by NIH R01HL115828 and R01HL133504. We would also like to thank General Electric, Inc., and the Irene D. Pritzker foundation for their financial and equipment support for this study.

\section{COMPETING INTERESTS}

All authors have no relevant financial or non-financial competing interests to report.

\section{AUTHOR CONTRIBUTIONS}

All authors have made significant contributions to this work and read and commented on previous manuscript versions. Jeesoo Lee performed echocardiography and MRI image-processing, statistical data analysis and drafted the first version of the manuscript. Nadia El Hangouche, Ashitha Pathrose, and Gilles Soulat participated in image processing and clinical data interpretation. Alex Barker was a senior investigator instrumental in study conceptualization and data acquisition management. James Thomas and Michael Markl were principal investigators who conceived this study and provided clinical and technical guidance of echocardiography and MRI data interpretation.

\section{ETHICS APPROVAL}

This study was performed in line with the principles of the Declaration of Helsinki. Approval was granted by the Internal Review Board of Northwestern University (STU00204434).

\section{CONSENT TO PARTICIPATE}

Informed consent was obtained from all individuals who participated in the study.

\section{CONSENT TO PUBLISH}

The authors affirm that human research participants provided informed consent to publish the study results.

\section{References}

1. Virani SS, Alonso A, Aparicio HJ et al. Heart disease and stroke statistics-2021 update: a report from the American Heart Association. Circulation. 2021;143(8):e254-e743.

2. Fedak PW, Verma S, David TE et al. Clinical and pathophysiological implications of a bicuspid aortic valve. Circulation. 2002;106(8):900-4. 
3. Michelena $\mathrm{HI}$, Khanna AD, Mahoney $\mathrm{D}$ et al. Incidence of aortic complications in patients with bicuspid aortic valves. JAMA. 2011;306(10):1104-12.

4. Girdauskas E, Borger MA, Secknus M-A et al. Is aortopathy in bicuspid aortic valve disease a congenital defect or a result of abnormal hemodynamics? A critical reappraisal of a one-sided argument. Circulation. 2011;39(6):809-14.

5. Bissell MM, Hess AT, Biasiolli L et al. Aortic dilation in bicuspid aortic valve disease: flow pattern is a major contributor and differs with valve fusion type. Circulation: Cardiovascular Imaging. 2013;6(4):499507.

6. Mahadevia R, Barker AJ, Schnell S et al. Bicuspid aortic cusp fusion morphology alters aortic threedimensional outflow patterns, wall shear stress, and expression of aortopathy. Circulation. 2014;129(6):673-82.

7. Mirabella L, Barker AJ, Saikrishnan N et al. MRI-based protocol to characterize the relationship between bicuspid aortic valve morphology and hemodynamics. Annals of biomedical engineering. 2015;43(8):1815-27.

8. Raghav V, Barker AJ, Mangiameli D et al. Valve mediated hemodynamics and their association with distal ascending aortic diameter in bicuspid aortic valve subjects. Journal of Magnetic Resonance Imaging. 2018;47(1):246-54.

9. Hope MD, Hope TA, Meadows AK et al. Bicuspid aortic valve: four-dimensional MR evaluation of ascending aortic systolic flow patterns. Radiology. 2010;255(1):53-61.

10. Dolan JM, Kolega J, Meng H. High wall shear stress and spatial gradients in vascular pathology: a review. Annals of biomedical engineering. 2013;41(7):1411-27.

11. Bollache E, Guzzardi DG, Sattari S et al. Aortic valve-mediated wall shear stress is heterogeneous and predicts regional aortic elastic fiber thinning in bicuspid aortic valve-associated aortopathy. The Journal of thoracic and cardiovascular surgery. 2018;156(6):2112-20. e2.

12. Meierhofer C, Schneider EP, Lyko $C$ et al. Wall shear stress and flow patterns in the ascending aorta in patients with bicuspid aortic valves differ significantly from tricuspid aortic valves: a prospective study. European Heart Journal-Cardiovascular Imaging. 2013;14(8):797-804.

13. Rodríguez-Palomares JF, Dux-Santoy L, Guala A et al. Aortic flow patterns and wall shear stress maps by 4D-flow cardiovascular magnetic resonance in the assessment of aortic dilatation in bicuspid aortic valve disease. Journal of Cardiovascular Magnetic Resonance. 2018;20(1):1-15.

14. Guzzardi DG, Barker AJ, Van Ooij P et al. Valve-related hemodynamics mediate human bicuspid aortopathy: insights from wall shear stress mapping. Journal of the American College of Cardiology. 2015;66(8):892-900. 
15. Baumgartner $\mathrm{H}$, Hung J, Bermejo $\mathrm{J}$ et al. Recommendations on the echocardiographic assessment of aortic valve stenosis: a focused update from the European Association of Cardiovascular Imaging and the American Society of Echocardiography. European Heart Journal-Cardiovascular Imaging. 2016;18(3):254-75.

16. De Paulis R, De Matteis GM, Nardi $P$ et al. Opening and closing characteristics of the aortic valve after valve-sparing procedures using a new aortic root conduit. The Annals of thoracic surgery. 2001;72(2):48794.

17. Leyh RG, Schmidtke C, Sievers H-H et al. Opening and closing characteristics of the aortic valve after different types of valve-preserving surgery. Circulation. 1999;100(21):2153-60.

18. Bazett HCJH. An analysis of the time relations of electrocardiograms. 1920;7:353-70.

19. Walker PG, Cranney GB, Scheidegger MB et al. Semiautomated method for noise reduction and background phase error correction in MR phase velocity data. Journal of Magnetic Resonance Imaging. 1993;3(3):521-30.

20. Berhane H, Scott M, Elbaz M et al. Fully automated 3D aortic segmentation of 4D flow MRI for hemodynamic analysis using deep learning. Magnetic resonance in medicine. 2020.

21. Potters WV, van Ooij P, Marquering $\mathrm{H}$ et al. Volumetric arterial wall shear stress calculation based on cine phase contrast MRI. Journal of Magnetic Resonance Imaging. 2015;41(2):505-16.

22. Gordon DZ, Abbasi MA, Lee J et al. Four-dimensional Flow Magnetic Resonance Imaging Quantification of Blood Flow in Bicuspid Aortic Valve. Journal of Thoracic Imaging. 2020;35(6):383-8.

23. Rahman O, Scott M, Bollache E et al. Interval changes in aortic peak velocity and wall shear stress in patients with bicuspid aortic valve disease. The international journal of cardiovascular imaging. 2019;35(10):1925-34.

24. Athar M, Ansari M, Khan M. Flow Characteristics of Elliptical Orifice Plates. ISH Journal of Hydraulic Engineering. 2003;9(2):22-35.

25. Flachskampf FA, Weyman AE, Guerrero JL et al. Influence of orifice geometry and flow rate on effective valve area: an in vitro study. Journal of the American College of Cardiology. 1990;15(5):1173-80.

26. Kamimura D, Hans S, Suzuki T et al. Delayed time to peak velocity is useful for detecting severe aortic stenosis. Journal of the American Heart Association. 2016;5(10):e003907.

27. Kim SH, Kim JS, Kim BS et al. Time to peak velocity of aortic flow is useful in predicting severe aortic stenosis. International journal of cardiology. 2014;172(3):e443-e6. 
28. van Ooij P, Markl M, Collins JD et al. Aortic valve stenosis alters expression of regional aortic wall shear stress: New insights from a 4-dimensional flow magnetic resonance imaging study of 571 subjects. Journal of the American Heart Association. 2017;6(9):e005959.

29. Fries $\mathrm{R}$, Graeter $\mathrm{T}$, Aicher $\mathrm{D}$ et al. In vitro comparison of aortic valve movement after valve-preserving aortic replacement. The Journal of Thoracic and Cardiovascular Surgery. 2006;132(1):32-7.

30. Higashidate M, Tamiya K, Beppu T et al. Regulation of the aortic valve opening: in vivo dynamic measurement of aortic valve orifice area. The Journal of thoracic and cardiovascular surgery. 1995;110(2):496-503.

31. Thubrikar M, Bosher LP, Nolan SP. The mechanism of opening of the aortic valve. The Journal of thoracic and cardiovascular surgery. 1979;77(6):863-70.

32. Bellhouse B, Bellhouse F. Mechanism of closure of the aortic valve. Nature. 1968;217(5123):86-7.

\section{Figures}




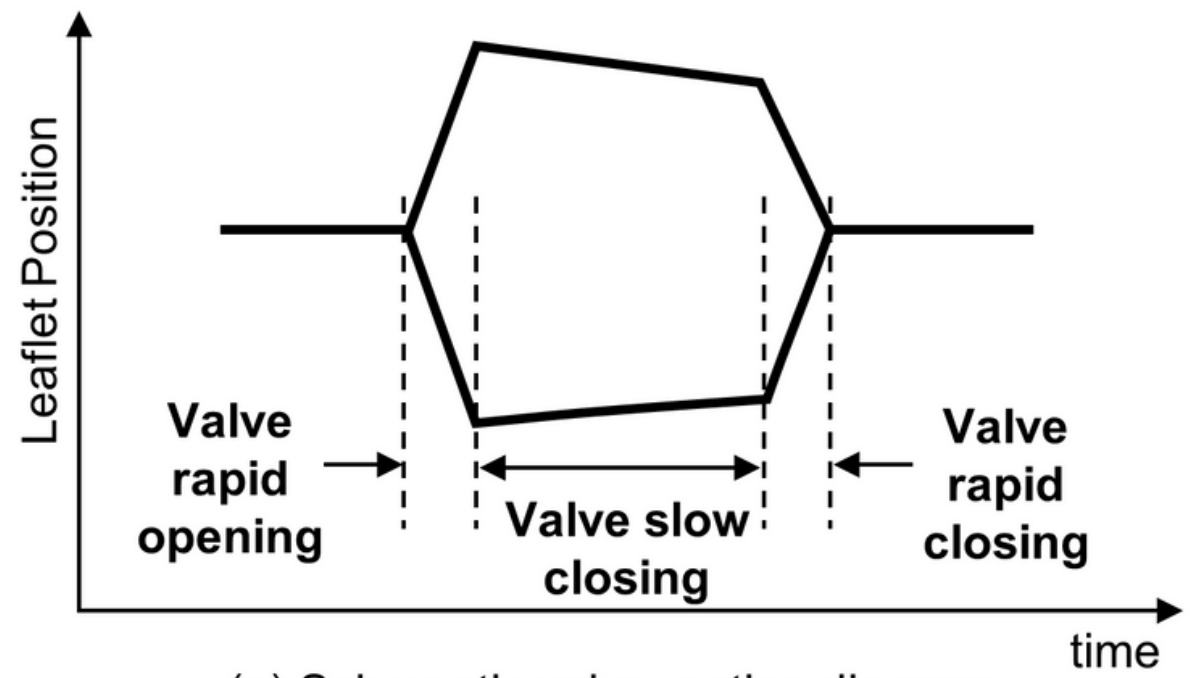

(a) Schematic valve motion diagram

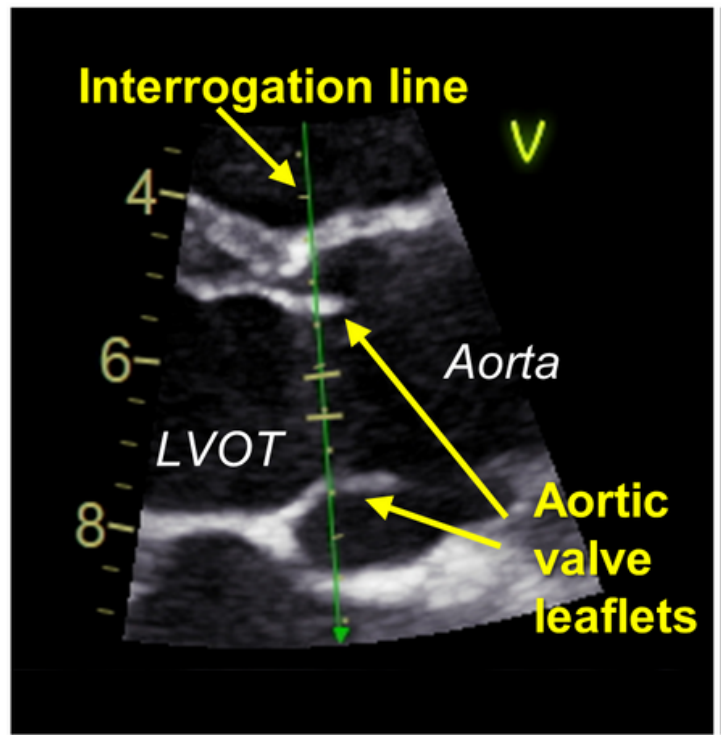

(b) Acquisition of valve motion diagram using B-mode

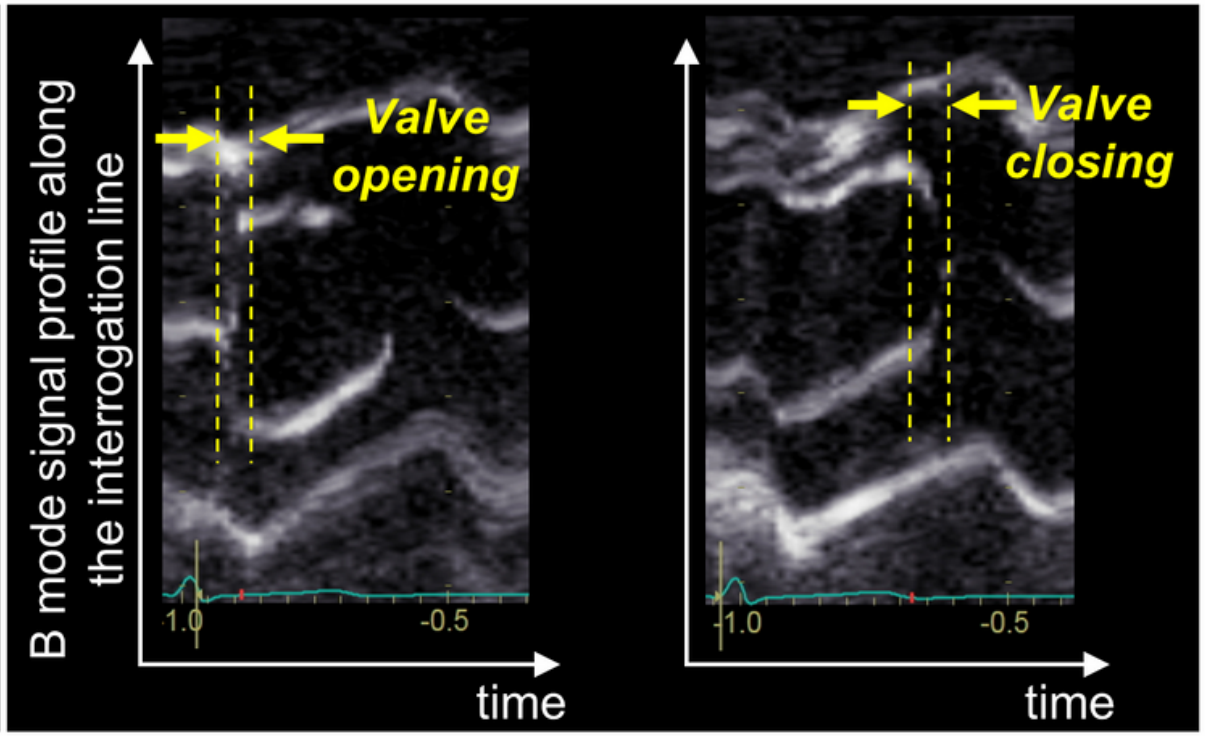

(c) Acquired valve motion diagram and timing measurement

Figure 1

Valve leaflet motion timing measurement. (a) A schematic diagram of aortic valve leaflet motion diagram. The diagram illustrates three distinct leaflet motion phases: rapid valve opening, slow closing, and rapid closing. (b) Reconstructing M-mode from 2D B-mode. The extraction of M-mode traces by placing an interrogation line (green line) across the valve leaflets. (c) Two valve leaflet motion diagrams are reconstructed by adjusting interrogation lines to capture the valve opening phase (left) and valve closing phase (right). 

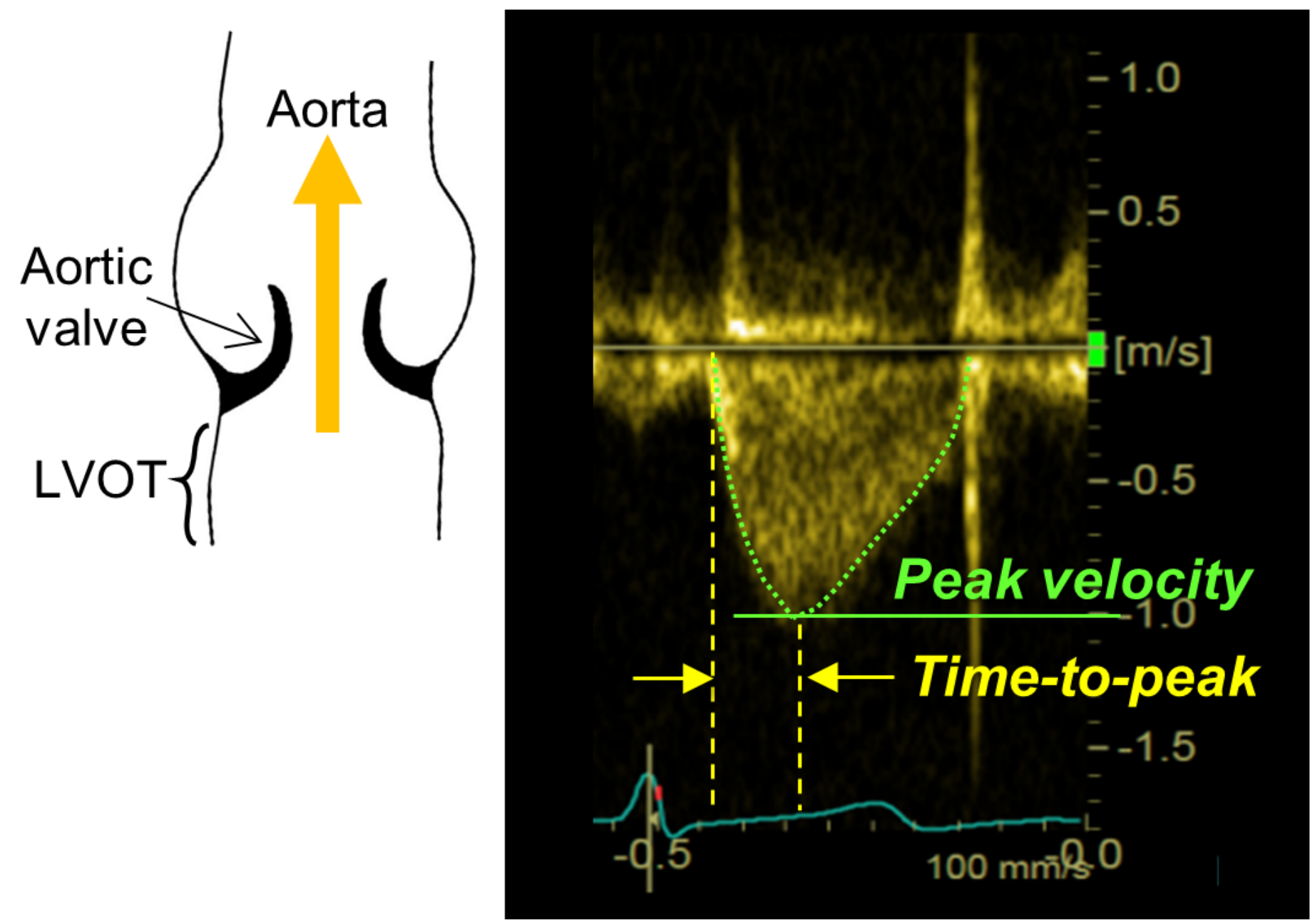

\section{Transvalvular flow velocity spectrum}

Figure 2

Transvalvular flow peak velocity and time-to-peak velocity measurements using aortic valve continuouswave Doppler. 


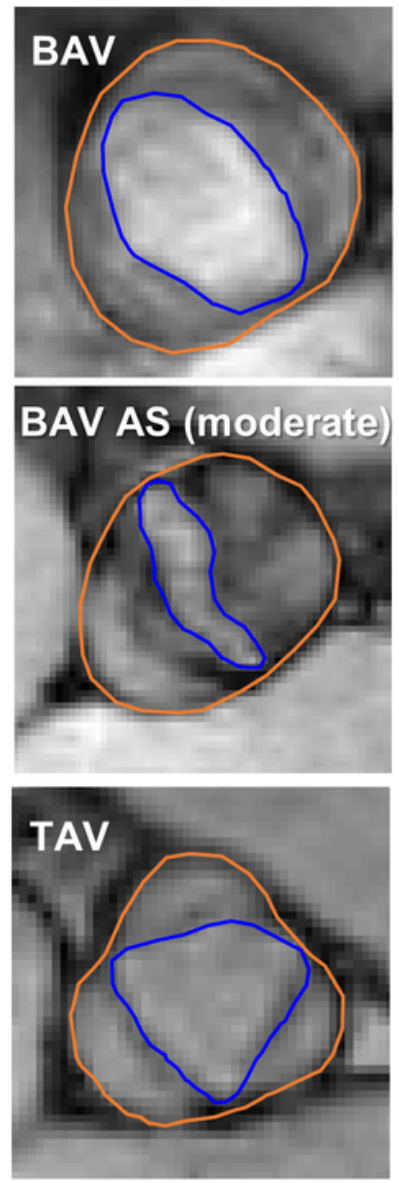

(a) Valve contours

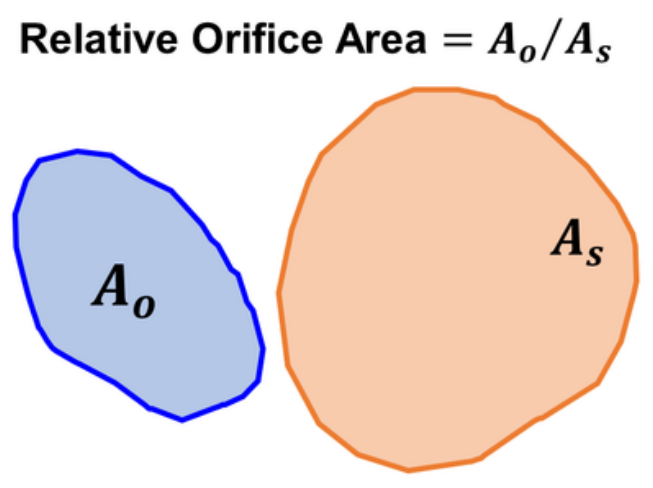

Aspect ratio $=d_{l} / d_{s}$

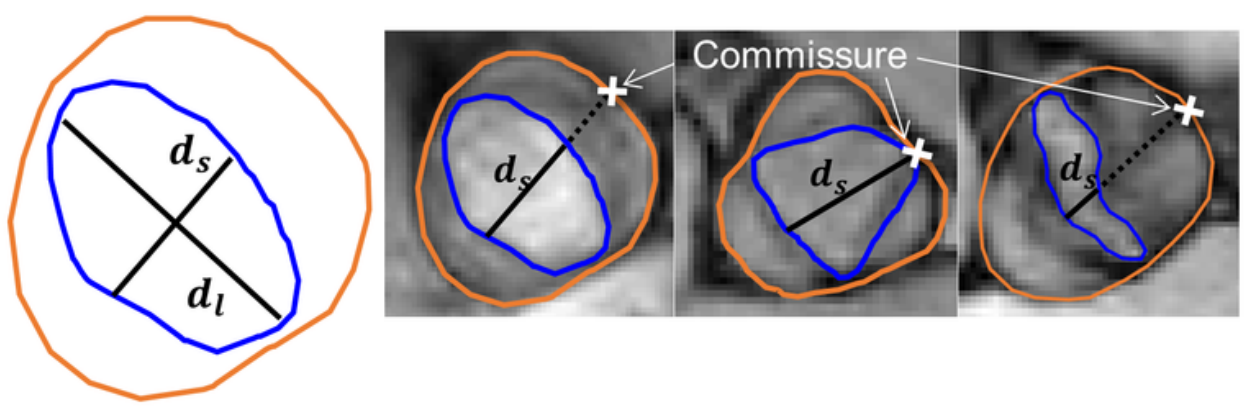

(b) Morphology characterization

Fig 3. Valve morphology characterization using $2 \mathrm{D}$ cine MRI at the level of the aortic valve. (a) Aortic sinus (orange) and valve orifice (blue) are contoured at the maximum valve opening. (b) $\boldsymbol{A}_{\boldsymbol{o}}$ and $\boldsymbol{A}_{\boldsymbol{s}}$ denote the valve orifice contour area and aortic sinus contour area, respectively. $\boldsymbol{d}_{\boldsymbol{l}}$ and $\boldsymbol{d}_{\boldsymbol{s}}$ denote the long and short axis length of the orifice contour, respectively. $\boldsymbol{d}_{\boldsymbol{s}}$ was measured based on a line from a commissural point of a conjoined leaflet on the sinus to the midpoint of the opposite leaflet. $\boldsymbol{d}_{\boldsymbol{l}}$ was automatically calculated based on the ellipse area formula $\left(d_{l}=4 A_{o} / \pi d_{s}\right)$.

\section{Figure 3}

See image above for figure legend

Figure 4 
Example maximum intensity projection images of peak systolic aortic wall shear stress (WSS) magnitude distribution. (a) a BAV patient without aortic stenosis, (b) a BAV patient with severe aortic stenosis. (C) a healthy control. The whilte closed curve denotes the ascending aortic region-of-interest (ROI).

\section{Figure 5}

Plots of significant association between echo and MRI parameters with and without BAV AS included in the analysis. Between valve morphology and hemodynamics: (a) orifice aspect ratio versus transvalvular peak velocity and (b) orifice aspect ratio versus maximum ascending aortic (AAo) wall shear stress (WSS). Between near-valve and distal hemodynamics: (C) Transvalvular peak velocity versus maximum AAo WSS. 